Wright State University

CORE Scholar

5-1-1995

\title{
Electrical Field Enhanced Thermal Quenching of a Prominent thermally Stimulated Current Peak in Semi-insulating GaAs
}

\author{
Z-Q. Fang
}

David C. Look

Wright State University - Main Campus, david.look@wright.edu

Follow this and additional works at: https://corescholar.libraries.wright.edu/physics

Part of the Physics Commons

\section{Repository Citation}

Fang, Z., \& Look, D. C. (1995). Electrical Field Enhanced Thermal Quenching of a Prominent thermally Stimulated Current Peak in Semi-insulating GaAs. Applied Physics Letters, 66 (22), 3033-3035.

https://corescholar.libraries.wright.edu/physics/52

This Article is brought to you for free and open access by the Physics at CORE Scholar. It has been accepted for inclusion in Physics Faculty Publications by an authorized administrator of CORE Scholar. For more information, please contact library-corescholar@wright.edu. 


\title{
Electrical field enhanced thermal quenching of a prominent thermally stimulated current peak in semi-insulating GaAs
}

\author{
Z-Q. Fang and D. C. Look \\ Physics Department, Wright State University, Dayton, Ohio 45435
}

(Received 6 February 1995; accepted for publication 6 April 1995)

\begin{abstract}
Detailed experimental results are presented for a "thermal quenching" of thermal stimulated current signals in the most prominent trap in undoped semi-insulating (SI) GaAs, $T_{5}$ with an activation energy of $0.27-0.31 \mathrm{eV}$. A possible model for the thermal quenching of $T_{5}$ is discussed, emphasizing the thermally stimulated nature of the quenching process, the effect of electric field and the formation of high-field domains. The thermal quenching of $T_{5}$ can frequency be observed in SI GaAs grown by the vertical gradient freeze (VGF) technique, or by the liquid encapsulated Czochralski (LEC) technique under certain conditions. (c) 1995 American Institute of Physics.
\end{abstract}

Thermally stimulated current (TSC) spectroscopy has long been used to study deep centers in undoped semiinsulating (SI) GaAs. ${ }^{1-6}$ In the TSC spectrum, six complete TSC peaks are usually observed; ${ }^{5}$ however, we often find a "thermal quenching" (TQ) of TSC signals in the most prominent peak $\left(T_{5}\right)$, i.e., a rapid drop of the TSC signal at a given temperature followed by a slow recovery at higher temperatures, often accompanied by attenuated current oscillations. In this letter, we present more detailed experimental results on the TQ and recovery processes and discuss the possible quenching mechanism.

In the typical TSC measurements, described elsewhere in detail, ${ }^{5,6} 1.45 \mathrm{eV}$ light with an intensity of $6 \times 10^{14}$ photon $/ \mathrm{cm}^{2} \mathrm{~s}$, from a $\frac{1}{4} \mathrm{~m}$ monochromator, was used to illuminate the sample at $83 \mathrm{~K}$ for $2.5 \mathrm{~min}$ under a bias of $18 \mathrm{~V}$. As an example, Fig. 1 shows differences in the $T_{5}$ signals in three adjacent samples which were cut along the diameter of a 3 in. VGF wafer (sample dimensions of $2 \times 6 \times 0.65$ $\mathrm{mm}^{3}$ ). In sample No 27 , a complete $T_{5}$ peak can be seen, while in sample No. 25, the TSC signals in the peak are quenched, resulting in nearly no observation of $T_{5}$. In contrast to the big difference in trap $T_{5}$ among the three samples, the traps $T_{2}$ and $T_{3}$ are quite similar. Here, we want to point out that although the peak heights of $T_{2}$ and $T_{3}$ are much lower than that of $T_{5}$, they are still important traps in SI GaAs and have been ascribed to $\mathrm{As}_{\mathrm{Ga}^{-}}$and $V_{\mathrm{As}}$-related point defects. ${ }^{6}$ Their lower peak heights are at least partially due to the lower carrier lifetime at their peak temperatures (180$230 \mathrm{~K}$ ), as indicated by a comparison of the photocurrents $\left(I_{\mathrm{ph}}\right)$ at 83 and $250 \mathrm{~K}$ (not shown), respectively.

To obtain further information on the TQ, we did TSC measurements on a LEC grown and multiple wafer annealed ${ }^{7}$ (MWA) sample, using different heating rates $\beta$ ( $\beta=0.05$ to $0.4 \mathrm{~K} / \mathrm{s}$ ), as shown in Fig. 2. Since we are dealing with the thermal emission of captured carriers from traps, a set of TSC measurements with different heating rates should show a shift in peak temperature for each trap. However, from each TSC spectrum in Fig. 2 we see shifts in both the quenching temperature $\left(T_{q}\right)$ and the recovery temperature ( $\left.T_{r}\right)$. This result implies that both quenching and recovery processes are controlled by some sort of thermally activated process. To confirm this suspicion, we checked the TQ in a different way. The sample was subjected to the same illumi- nation, and a thermal scan with $\beta=0.3 \mathrm{~K} / \mathrm{s}$ was then performed in such a way that it stopped at a temperature $T_{w}$ (e.g., 115, 120, or $125 \mathrm{~K}$ ) below the temperature at which the TQ would have occurred $(\sim 130 \mathrm{~K})$. The results are shown in Fig. 3(a). This procedure was followed by waiting a time $t_{w}$, which depended on the selected $T_{w}$, then fast cooling down to $83 \mathrm{~K}$, and finally heating the sample in a regular thermal scan $(\beta=0.3 \mathrm{~K} / \mathrm{s})$; these data are shown in Fig. 3(b). From Fig. 3(a), we note that for each chosen $T_{w}$, the initial slow signal decay, which is due to the thermal emission of capture carriers from the region of traps $T_{5}$ and $T_{5}^{*}$, is followed by a TQ of more than two orders of magnitude. The initial delay time depends of course on the $T_{w}$, since the higher the $T_{w}$, the higher the carrier emission rate for the trap. These results indicate that the TQ is indeed partially or fully related to a thermally activated process, i.e., the thermal release of captured carriers from the region of traps $T_{5}^{*}$ and $T_{5}$. From Fig. 3(b), we find that after the TQ occurs for each $T_{w}$, no TSC signals can be observed at $T<140 \mathrm{~K}$, but the other traps at $T>140 \mathrm{~K}$, i.e., $T_{2}, T_{3}$, and $T_{4}$ are not affected at all. This result means that the TQ of $T_{5}$ is not associated with other traps, at least not those TSC traps occurring above $140 \mathrm{~K}$.

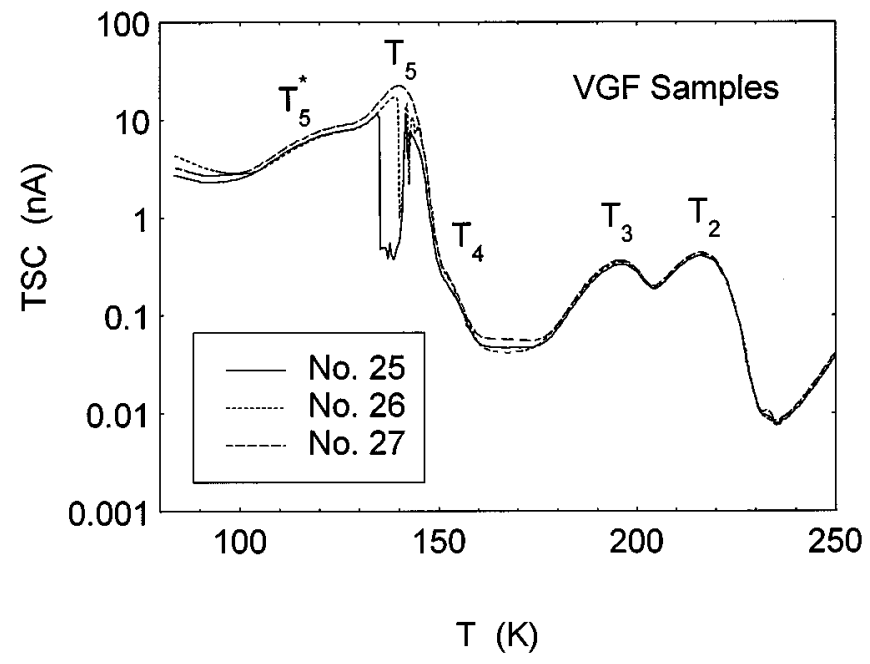

FIG. 1. TSC spectra for three adjacent samples cut along the diameter of a 3 in. VGF wafer. 


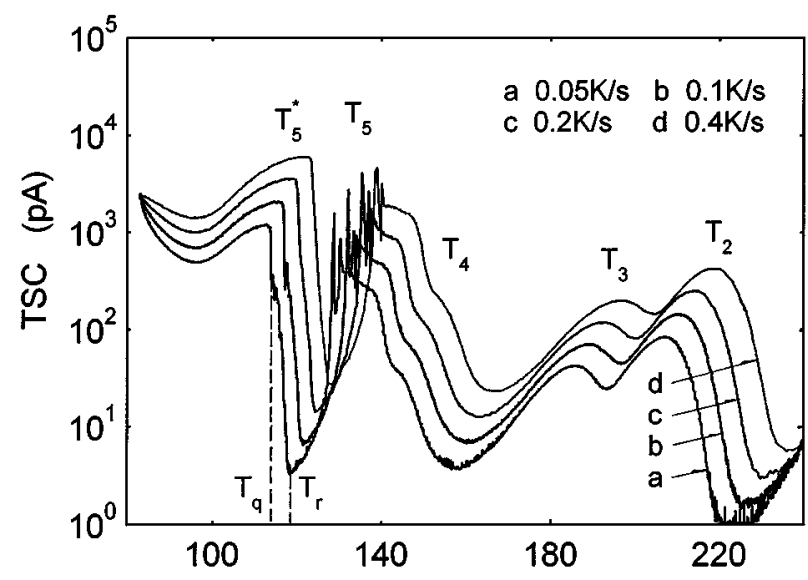

$T(K)$

FIG. 2. TSC spectra, including TQ of $T_{5}$, as a function of heating rates $\beta$ ( $\beta=0.05$ to $0.4 \mathrm{~K} / \mathrm{s}$ ) for a MWA sample.

Next, we present the bias voltage effect on the TQ. A set of TSC spectra for the MWA sample, using the same excitation conditions at $83 \mathrm{~K}$ but various bias voltages from 1.5 to $36 \mathrm{~V}$, is shown in Fig. 4; the photocurrent at $83 \mathrm{~K}$ versus bias is shown in the inset. From this figure we see that the TQ of $T_{5}$ is closely related to the applied bias or the electrical field $\mathscr{E}$; i.e., at $\mathscr{E}<7.5 \mathrm{~V} / \mathrm{cm}$, a complete $T_{5}$ can be measured, whereas at $\mathscr{E}>15 \mathrm{~V} / \mathrm{cm}$ the TQ occurs, with the threshold temperature $T_{q}$ decreasing as $\mathscr{E}$ increases. For this sample we can find a threshold electrical field $\mathscr{E}_{\text {th }}$ of about $13 \mathrm{~V} / \mathrm{cm}$, at which the TQ of $T_{5}$ begins to appear. The photocurrent shows a non-ohmic behavior at $\mathscr{E}>15 \mathrm{~V} / \mathrm{cm}$, which is very similar to the results of earlier studies on the photoelectronic properties of high-resistivity GaAs:O and $\mathrm{GaAs}: \mathrm{Cr}$ by Lin et al. ${ }^{8,9}$ In contrast to the photocurrent, the TSC for all traps, except for $T_{5}$, and the dark current (at $T>250 \mathrm{~K}$ where it is measurable) show good ohmic behavior. Thus, the TQ of $T_{5}$ is evidently connected with the photocurrent saturation.

Before we proceed further, it is helpful to summarize what we know about $T_{5} . T_{5}$ is closely related to EL2 or $\mathrm{As}_{\mathrm{Ga}}$, based mainly on its IR quenching behavior ${ }^{4}$ and its thermal recovery, with a rate $r=2.0 \times 10^{8} \exp (-0.26$ $\mathrm{eV} / \mathrm{kT}) \mathrm{s}^{-1}{ }^{5}$. From TSC measurements, using a Schottky contact and surface absorbed light $(1.96 \mathrm{eV}), T_{5}$ is judged by its variation with bias to be a hole trap,${ }^{10}$ but it might also trap electrons. From a study of molecular beam epitaxial GaAs grown at $200-250{ }^{\circ} \mathrm{C}$, which is thought to have abundant concentrations of $\mathrm{As}_{i}, \mathrm{As}_{\mathrm{Ga}}$, and $V_{\mathrm{Ga}}$, it was concluded that $T_{5}$ must involve $V_{\mathrm{Ga}}$, because, of these three primary defects, only $V_{\mathrm{Ga}}$ is expected to have acceptor levels below midgap. ${ }^{11}$ The trapping and recombination of free carriers by $T_{5}$, studied by Desnica et al., ${ }^{12}$ indicates that $T_{5}$ (labeled $T_{3}$ in Ref. 12) behaves more like a recombination center than an ordinary electron trap, since its capture cross section for holes can be larger than that for electrons. Thus, we can infer that $T_{5}$ is probably a $V_{\mathrm{Ga}}$-related hole trap.

The thermal quenching (TQ) model for $T_{5}$ must explain the following observations: (a) the abruptness of the drop in TSC at temperature $T_{q}$; (b) the dependence of $T_{q}$ on heating
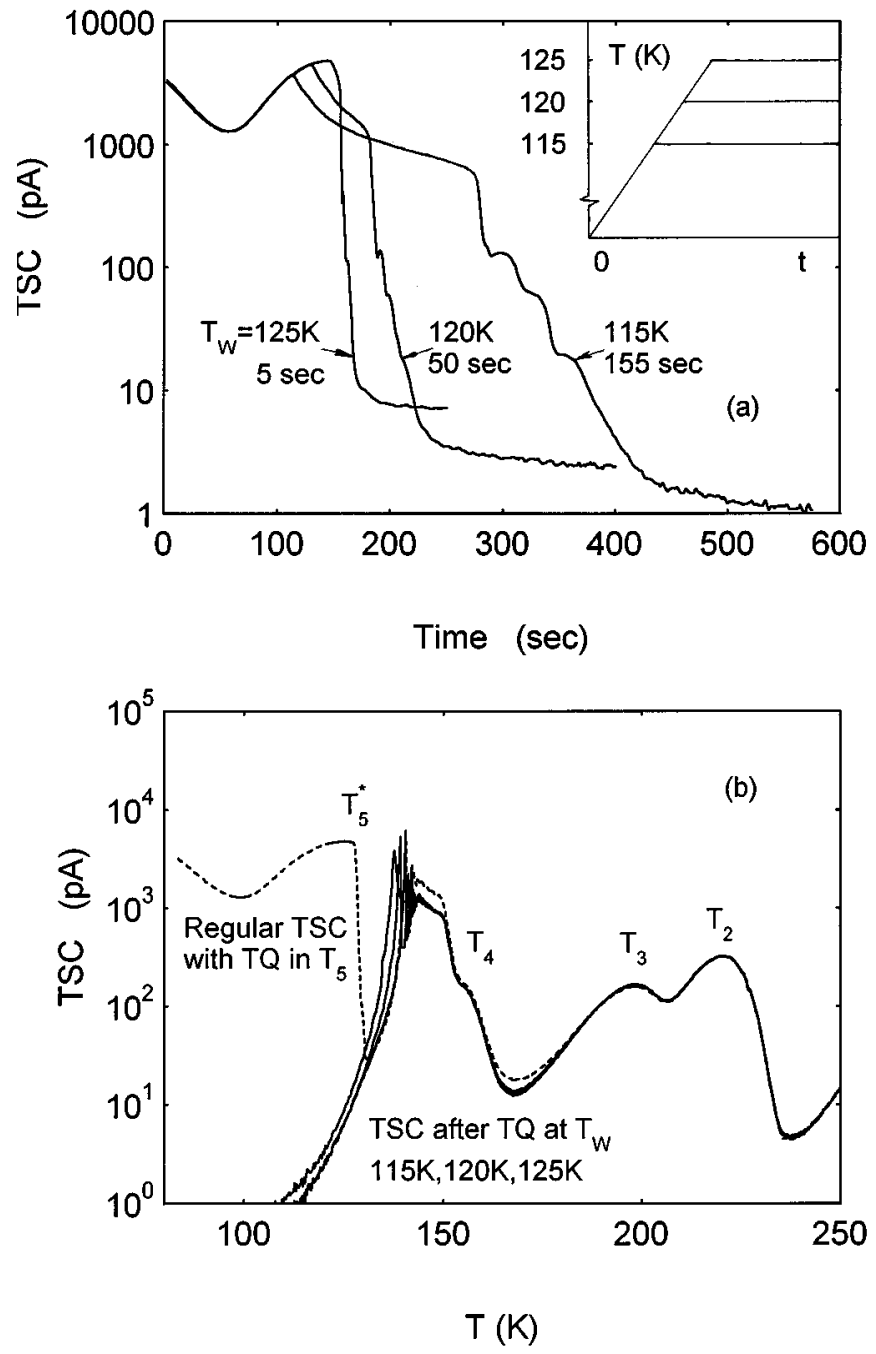

FIG. 3. Observation of TQ in $T_{5}$ on a MWA sample using an alternative method: (a) TQ begins at a certain decay time depending on the waiting temperature $\left(T_{w}\right)$, as shown in the thermal scan schedule in the inset; (b) TSC spectra measured after the waiting time and after fast cooling the sample to $83 \mathrm{~K}$; also shown is a regular TSC spectrum with TQ of $T_{5}$ as in Fig. 2.

rate $\beta$ and electric field $\mathscr{E}$; (c) the small value of the threshold electric field $\mathscr{E}_{\text {th }}$ necessary to cause the TQ; and (d) the saturation of photoconductivity at $\mathscr{E}>\mathscr{E}_{\text {th }}$. Observation (a), the abruptness of the TSC drop, suggests a cooperative process, i.e., one in which the process rate increases as the process continues. Such an event can occur in SI GaAs because $\mathrm{EL}_{2}{ }^{+}$and probably other $\mathrm{As}_{\mathrm{Ga}}$-related defects have barriers for electron capture, so that higher-energy electrons are more easily captured. Then, if part of a sample somehow attains a higher electric field $\mathscr{E}$, perhaps due to an instability, more of the electrons in that region will be captured, leading to a higher resistivity. The higher resistivity will now produce an even higher electric field (greater voltage drop) and the process will speed up, eventually creating a high-field, highresistivity domain that will greatly decrease the sample current. This charged domain will eventually drift to the opposite electrode and there be annihilated, thus increasing the current. Current oscillations, commonly seen in SI GaAs, are usually attributed to the periodic formation and annihila- 


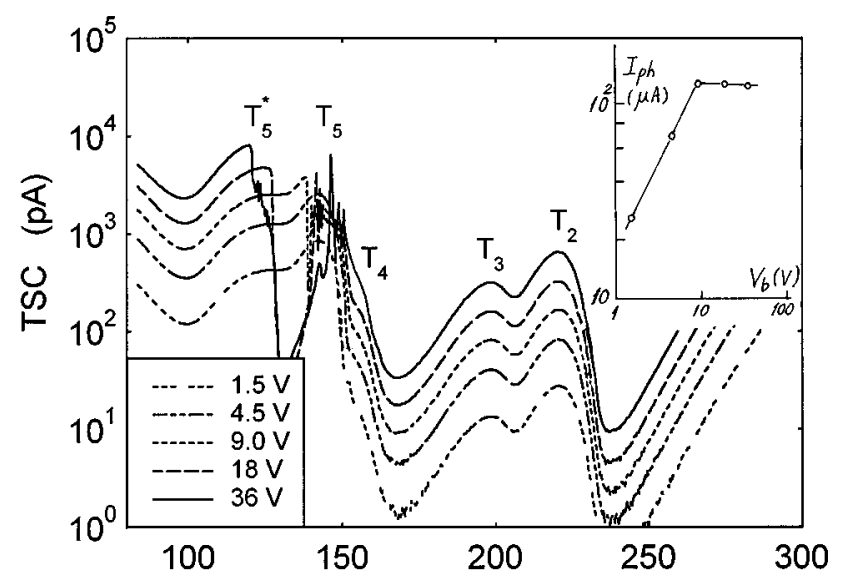

$T(K)$

FIG. 4. TSC spectra, including TQ of $T_{5}$, as a function of applied bias $\left(V_{b}\right)$ for a MWA sample, with $I_{\mathrm{ph}}$ at $83 \mathrm{~K}$ vs $V_{b}$ shown in the inset.

tion of domains. However, it should be noted that the domain drift may be slow, and the domain may appear to be stationary over a certain time period. The data in this report are consistent with the formation of domains. For example, we would expect such a formation to take longer at lower current levels, as indeed is seen in Fig. 2. For $\beta=0.05 \mathrm{~K} / \mathrm{s}$, there are approximately $340 \mathrm{~s}$ from the current minimum at $T=97$ $\mathrm{K}$ to the quenching temperature $T_{q}=114 \mathrm{~K}$, while for $\beta=0.4 \mathrm{~K} / \mathrm{s}$, the time from the minimum to $T_{q}$ is about $70 \mathrm{~s}$. This ratio of quenching times is close to the inverse of the ratio of the currents at $T_{q}$. The same qualitative picture holds in Fig. 3(a), in which a lower current (as well as a lower electron energy) at the lower waiting temperature would lead to a longer time before quench. The dependence on electric field is seen in Fig. 4, which illustrates that higher $\mathscr{E}$ leads to an earlier quench. In spite of these aspects of the present quenching process, which appear similar to those previously observed in SI GaAs, ${ }^{13,14}$ there are also some differences. For example, threshold fields for both photocurrent ${ }^{13}$ and $\mathrm{TSC}^{14}$ were found to be at least $500 \mathrm{~V} / \mathrm{cm}$ in the previous works, whereas we see TSC quenching effects at threshold fields as low as $15 \mathrm{~V} / \mathrm{cm}$. It is perhaps significant that most of the previous studies were performed at temperatures above $140 \mathrm{~K}$, so that $\mathrm{As}_{\mathrm{Ga}}$-related defects were always in their normal states. However, we believe that the data in Fig. 3(b) indicate that the defects involved in our fieldenhanced trapping process may be $\mathrm{As}_{\mathrm{Ga}}$-related defects in their metastable states. The reason is that the recovery of the thermally quenching TSC exactly follows the recovery of the optically quenched TSC, and the latter is known to have the same kinetics as the EL2 (or $\mathrm{As}_{\mathrm{Ga}}$ ) recovery from metastability. Thus, $\mathrm{As}_{\mathrm{Ga}}$ metastability seems to be involved in the present TQ. Note also, from the inset of Fig. 4, that the photocurrent $(\mathrm{PC})$ at $83 \mathrm{~K}$ saturates at electric fields that are high enough to cause TSC quenching. The PC does not itself quench, probably because $1.45-\mathrm{eV}$ excitation continuously provides electrons from the valence band, rather than just from EL2 and traps such as $T_{5}$. The PC saturation could be caused by a reduced lifetime $\tau$ due to the increased ability of the photoexcited electrons to surmount the $\mathrm{As}_{\mathrm{Ga}}^{+}$capture barrier. For example, if $\tau \propto \mathscr{E}^{-1}$ for $\mathscr{E}>\mathscr{E}_{\text {th }}$, then the PC would saturate at $\mathscr{E}_{\text {th }}$. However, the saturation could also be explained by a high-field, high-resistivity region which increases in size as $\mathscr{E}$ increases, as, e.g., occurs in a field-effect transistor.

In summary, we suggest that the thermal quenching of the TSC in the region of $T_{5}$ can be explained by the formation of a current-limiting, high-field domain that is nearly stationary (since large oscillations do not appear until $T_{5}$ has recovered). The domain could form when a high-electricfield instability in a particular region begins to cause fieldenhanced trapping of the TSC electrons in that region, thus causing even higher fields and further trapping. The highfield, high-resistivity domain thus formed could quickly limit current, as is observed. Because the recovery of the TSC follows a temperature dependence exactly the same as that found for metastable EL2 and other $\mathrm{As}_{\mathrm{Ga}}$-related defects, it is likely that the trapping centers are themselves $\mathrm{As}_{\mathrm{Ga}}$ related. We have previously proposed that $T_{5}$ itself is an $\mathrm{As}_{\mathrm{Ga}}$-related defect, perhaps $\mathrm{As}_{\mathrm{Ga}}-V_{\mathrm{Ga}} \cdot{ }^{11}$ This defect could trap an electron during the illumination at $83 \mathrm{~K}$ and then release that electron to the conduction band at higher temperatures, i.e., the normal TSC process. However, at high electric fields, it might be possible for the electron to immediately be recaptured at the $\mathrm{As}_{\mathrm{Ga}}$ component of the defect, and cause a metastable transition of the $\mathrm{As}_{\mathrm{Ga}}$. Above $140 \mathrm{~K}$, the $\mathrm{As}_{\mathrm{Ga}}$ would return to its normal state, and the electron could finally be emitted to the conduction band. This model is consistent with the thermal quenching being triggered by an emission process with an activation energy close to that of $T_{5}$, as is found from an analysis of the $T_{q}$ vs $\beta$ data in Fig. 2. However, further evidence is necessary before a microscopic TQ model can be firmly established.

The work of Z-Q.L. and D.C.L. was supported under USAF Contract No. F33615-91-C-1765.

${ }^{1}$ D. I. Desnica, J. Electron. Mater. 21, 463 (1992).

${ }^{2}$ J. P. Fillard, J. Bonnafe, and M. Castagne, Solid State Commun. 52, 855 (1984).

${ }^{3}$ U. V. Desnica, D. I Desnica, and B. Santic, Appl. Phys. Lett. 58, 278 (1991).

${ }^{4}$ Z-Q. Fang and D. C. Look, Appl. Phys. Lett. 59, 48 (1991).

${ }^{5}$ Z-Q. Fang and D. C. Look, J. Appl. Phys. 73, 4971 (1993).

${ }^{6}$ Z-Q. Fang and D. C. Look, J. Appl. Phys. 69, 8177 (1991).

${ }^{7}$ M. Mori, G. Kano, T. Inoue, H. Shimakura, H. Yamamato, and O. Oda, in Semi-insulating III-V Materials, Toronto, 1990, edited by A. G. Milnes and C. J. Miner (Adam Hilger, Bristol, 1991), p. 155.

${ }^{8}$ A. L. Lin, E. Omelianovski, and R. H. Bube, J. Appl. Phys. 47, 1852 (1976).

${ }^{9}$ A. L. Lin and R. H. Bube, J. Appl. Phys. 47, 1859 (1976).

${ }^{10}$ Z-Q. Fang, L. Shan, T. E. Schlesinger, and A. G. Milnes, Mater. Sci. Eng. 5, 397 (1990).

${ }^{11}$ Z-Q. Fang and D. C. Look, Appl. Phys. Lett. 63, 219 (1993).

${ }^{12}$ U. V. Desnica, B. Santic, D. I. Desnica, and M. Pavlovic, J. Electron. Mater. 22, 403 (1993).

${ }^{13}$ D. A. Johnson, R. A. Puechner, and G. N. Maracas, J. Appl. Phys. 67, 300 (1990).

${ }^{14}$ M. Kaminska, J. J. Parsey, J. Lagowski, and H. C. Gates, Appl. Phys. Lett. 41, 989 (1982). 